\title{
Estado del conocimiento del conflicto por grandes felinos y comunidades rurales en Colombia: avances y vacíos de información
}

\author{
Irene Aconcha-Abril \\ Proyecto de Conservación de Aguas y Tierras - ProCAT Colombia \& Universidad Nacional de Córdoba, \\ Rondeau 798 X5000AVP, Córdoba, Argentina. ireneaconcha@gmail.com \\ J. Sebastián Jiménez-Alvarado \\ Catalina Moreno-Díaz C \\ Diego A. Zárrate-Charry \\ José F. González-Maya \\ Proyecto de Conservación de Aguas y Tierras - ProCAT Colombia \& Instituto de Ecología, Universidad \\ Nacional Autónoma de México, México.
}

Desde los inicios de la civilización, la convivencia entre humanos y carnívoros se ha dado de manera competitiva por espacio y recursos. Sin embargo, en la actualidad esta convivencia se ha transformado principalmente en conflicto, cuando los depredadores se ven obligados a coexistir con animales domésticos en paisajes dominados por el hombre (Pitman et al. 2002). Aunque la depredación es un proceso fundamental para el mantenimiento de la biodiversidad y los procesos ecológicos, el concepto se ha transformado, debido a las malas percepciones locales y el manejo inadecuado de la información, donde los depredadores, en especial los grandes felinos se perciben como amenazas sobre la seguridad de las personas y sus propiedades (Márquez \& Golstein 2014, Inskip \& Zimmermann 2009). Aunado a esto, los eventos de conflicto han incrementado en las últimas décadas debido a presiones directas sobre los carnívoros, que incluyen la reducción de sus hábitats naturales, el crecimiento demográfico humano, el incremento en la cacería retaliativa e incidental por parte de los pobladores rurales afectados, la disminución de las poblaciones de presas naturales, y el manejo inadecuado de las actividades antrópicas, entre otros factores (Hoogesteijn and Hoogesteijn 2014, González-Maya et al. 2013, Michalski et al. 2006), problemáticas que afectan al 75\% de las especies de felinos del mundo (Inskip \& Zimmermann 2009).

La principal causa de reducción de felinos en América Latina, que afecta principalmente a especies de gran porte como el Jaguar y el Puma (Azevedo and Murray 2007, Michalski et al. 2006), se atribuye en gran medida al conflicto ocasionado por los ataques sobre animales domésticos. En general, este problema se convierte en una situación de alta preocupación para muchos productores, y particularmente para los campesinos, que se traduce en pérdidas económicas considerables, incluso cuando estas pueden ser relativamente bajas en comparación con otros problemas asociados a la tenencia y cuidado de animales (i.e. Enfermedades, abortos, muertes accidentales, etc.; González-Maya et al. 2013).

En Colombia, un país naturalmente forestal, y con una larga historia de deforestación y transformación de áreas para crianza de animales domésticos, que a la vez hace parte de la distribución natural de grandes felinos en una amplia proporción, no se escapa a esta realidad. En el país tanto histórica como actualmente se registran numerosos ataques, tanto documentados como no documentados, sobre ganado y animales domésticos, principalmente atribuidos al Jaguar (Panthera onca), al Puma (Puma concolor), al Oso de Anteojos (Tremarctos ornatus) y por perros asilvestrados (Canis lupus familiaris); estos últimos en donde los ataques son comúnmente confundidos con los ataques de grandes felinos (García-Villareal 2014). Dada esta antagonista relación histórica entre producción y grandes depredadores ha conducido en general a una percepción negativa de los últimos, lo que generalmente se traduce en amenazas serias para su conservación.

A pesar de ser un fenómeno histórico, en años recientes la frecuencia de ocurrencia de eventos de depredación conflicto ha venido en aumento, dados los múltiples procesos sociales y de manejo de la tierra, que de cierta manera han promovido este

\footnotetext{
Mammalogy Notes | Notas Mastozoológicas

Sociedad Colombiana de Mastozoología

Vol. 3 Num. 1| 2016
} 
aumento, o por lo menos la visibilización del mismo. Dada la magnitud de esta amenaza, el Programa Nacional de Conservación de Felinos de Colombia propuesto por el Ministerio de Ambiente y Desarrollo Sostenible (MAVDT y FVSN 2007), entre otros documentos, incluyó el conflicto como uno de los principales temas a trabajar con el fin de reducir el efecto de esta amenaza sobre las poblaciones silvestres. Así mismo, a niveles regionales esta problemática también ha sido claramente identificada (e.g., Plan de Conservación de los Felinos del Caribe Colombiano; Castaño-Uribe et al. 2013), por lo que actualmente se considera una prioridad de acción e investigación a nivel nacional.

A pesar de estas acciones y el gran número de casos reportados en el país, el conocimiento que se tiene sobre esta problemática en Colombia es mínimo y en muchos de los casos los ataques no se encuentran documentados en gran parte por los trámites ineficientes de las entidades competentes. Adicionalmente, los pocos estudios publicados están consolidados dentro de literatura gris, o son de difícil acceso o no tienen impacto sobre planificación o manejo de la amenaza. De esta manera, conocer el panorama actual y los vacíos de información se vuelve una pieza clave para iniciar acciones que promuevan el manejo preventivo y correctivo del conflicto.

En Colombia la problemática ha venido incrementando con el tiempo, contrario a la información relacionada con el tema, donde hasta la fecha el estudio de los conflictos ha sido abordado principalmente desde perspectivas ecológicas y sociales. De un total de 25 documentos (Bibliografía S1), la mayor proporción se han realizado para la zona Andina, fueron publicados a partir del 2007 (Figura 1A), la mayoría se encuentran en literatura gris (Figura 1B), la mayoría sobre jaguar y puma (Figura 1D), y los temas específicos de los documentos incluyen desde generalidades hasta caracterizaciones, diagnósticos y propuestas de solución (Figura 1C).
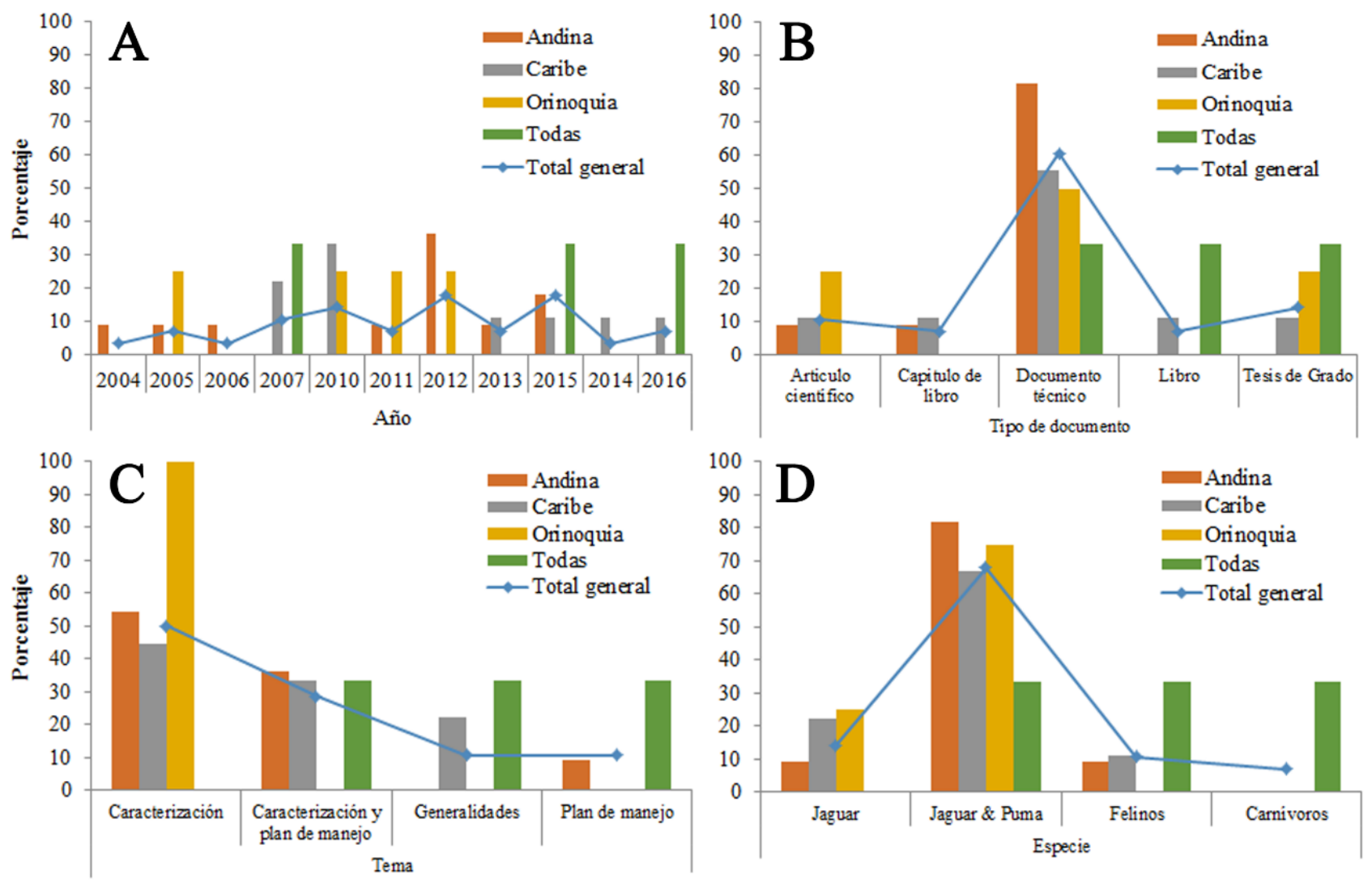

Figura1. Distribución de los documentos existentes sobre conflicto en el país según (A) año, (B) Tipo de documento, (C) Tema y (D) especie.

\section{Mammalogy Notes | Notas Mastozoológicas \\ Sociedad Colombiana de Mastozoología \\ Vol. 3 Num. 1| 2016}


La escasa documentación que existe a nivel nacional en torno al conflicto por grandes felinos y las comunidades rurales son el reflejo de los vacíos de información a nivel local, regional y nacional, donde la gran mayoría de estudios existentes responden a necesidades o requerimientos específicos de las Corporaciones Autónomas Regionales. Estos han estado enfocados en caracterizar el conflicto mediante seguimiento de los ataques y en algunos casos proponer medidas de manejo a nivel local. Sin embargo, estos estudios se realizan y publican a nivel interno de las mismas instituciones, generalmente como informes técnicos, y sus resultados no son divulgados a la comunidad científica.

Los estudios más completos corresponden a aquellos que incluyen la caracterización en campo, el diagnostico social, la distribución espacial de los ataques y la propuesta con mecanismos para la prevención y mitigación de estos eventos. A pesar de esto, ninguno de los documentos cuenta con reportes de experiencias de manejo exitosas que se hayan realizado en el país. La región Andina, Caribe y Orinoquia son las únicas regiones que reportan conflicto y se relacionan con las zonas que potencialmente presentan mayor probabilidad de conflicto (Aconcha 2016). Sin embargo, la región Pacifica y Amazonia no cuentan con ningún tipo de estudio o análisis que brinde un panorama de la situación dentro de las mismas.

A pesar de que el Jaguar está catalogado como el felino más conflictivo de Latinoamérica (Zimmermann et al 2010), el Puma, al contar con un rango de distribución y amplitud de nicho ecológico mayor, debe considerarse dentro de los estudios que diagnostiquen el conflicto, puesto que la comunidad siempre identifica un único depredador. Sumado a esto, muchos de los reportes no tienen claridad sobre el depredador en cuestión y en la mayoría de casos, la localidad de ocurrencia se ubica dentro del rango de distribución de ambos felinos; por esta razón es importante la inclusión tanto del Jaguar como del Puma dentro de las caracterizaciones e investigaciones en campo.

Dentro de las principales necesidades es importante convocar a las Corporaciones Autónomas Regionales e instarles jugar un papel dentro de los retos y desafíos que implica ampliar el conocimiento de los conflictos por grandes felinos a nivel nacional. Así mismo, estas deben promover la sistematización de los reportes de ataques y de muertes de grandes felinos como el insumo principal para la generación de conocimiento, el cual mediante la caracterización (e.g.. in situ, social) y la participación de las comunidades rurales afectadas dentro del proceso, permitirán la ejecución de planes de manejo aplicables a cada región. Las alianzas con otras instituciones (e.g. Organizaciones no Gubernamentales, entidades privadas) puede aportar al éxito del proceso desde diferentes perspectivas (e.g. sociales, ambientales, económicas, técnicas, etc.); adicionalmente, la divulgación de resultados, experiencias y conclusiones puede ser el aporte más valioso para el enriquecimiento de la información referente al conflicto asociado a grandes felinos y comunidades rurales en Colombia.

Dentro de la situación actual, la generación de conocimiento es una prioridad para conocer y comprender la dinámica de los conflictos por grandes felinos sobre los animales domésticos y su afectación sobre las comunidades rurales; información que es necesario incluya el entendimiento de las causas que lo generan, el análisis de las consecuencias sobre las comunidades humanas afectadas, las poblaciones de félidos y sus dinámicas con los ecosistemas en un contexto local y regional.

Basados en el conocimiento actual, consideramos que los principales vacíos sobre el tema son:

1. Magnitud de conflicto con especies distintas al Jaguar y Puma en las regiones Andina, Caribe y Orinoquía.

2. Magnitud del conflicto con felinos en todas las zonas aledañas a remanentes de unidades forestales en el país.

3. Identificación de presencia de conflicto entre pobladores locales y felinos en la región Amazonía y Chocó.

4. Efectos de liberación de mesodepredadores en zonas donde la caza por retaliación ha afectado las poblaciones naturales de grandes felinos.

5. Impacto económico de los ataques a animales domésticos por felinos.

6. Manejo de animales en unidades agropecuarias de hatos menores a 50 animales.

7. Variables a escala local y regional que potencian el conflicto en las distintas regiones del país, con un énfasis en la región Andina, Caribe y Orinoquía.

8. Distribución, ecología y patrones de movimiento de carnívoros que actualmente presentan situaciones de conflicto con unidades agropecuarias.

\footnotetext{
Mammalogy Notes | Notas Mastozoológicas

Sociedad Colombiana de Mastozoología

Vol. 3 Num. 1| 2016
} 
9. Ecología poblacional de grandes carnívoros con énfasis en felinos y requerimientos de hábitat para mantener poblaciones viables.

10. Análisis poblacional de presas y su relación con el uso por parte de las comunidades locales.

11. Estrategias económicas rurales compatibles con la presencia de grandes carnívoros.

12. Prácticas de reducción de conflicto de bajo costo para implementar en unidades agropecuarias.

Así mismo, las prioridades propuestas de investigación y acción incluyen:

1. Realizar los primeros levantamientos de información sobre situaciones de conflicto en las regiones Amazonía y Chocó, ya que son zonas de distribución y hábitat de Jaguar y Puma pero se desconoce el estado actual de conflicto o la existencia del mismo.

2. Construir un sistema integrado de reportes que pueda ser usado a nivel nacional, y que se fundamente en visitas técnicas que permitan discernir en los ataques y conocer si fue realizado por alguna especie de felino u otro carnívoro.

3. Identificar medidas de reducción y prevención de ataques, diseñados de manera específica hacia distintas unidades productivas agropecuarias de distintos tamaños.

4. Promover el estudio y crecimiento de unidades agrícolas compatibles con las necesidades ecológicas de los carnívoros, con el fin de reducir el impacto en el tamaño de su hábitat.

5. Incentivar mecanismos de pagos por productos, o por servicios de biodiversidad a propietarios de predios que mantengan parches de bosque dentro de zonas de hábitat o conectividad de grandes carnívoros.

6. Evaluar posibles esquemas de pagos por pérdida de animales en zonas prioritarias de hábitat de carnívoros, asociados a bancos de animales.

7. Construir protocolos de atención a situaciones de conflicto que incluyan la participación de todas las entidades asociadas al manejo de fauna en los departamentos (Corporaciones, policía, ejercito, jueces ambientales, defensoría y contraloría).

8. Generar espacios de discusión entre las comunidades y las entidades estatales de modo que se entienda plenamente la ley en relación a la caza de felinos y sus presas.

9. Fortalecer las medidas de seguimiento y judicialización en caso de caza por retaliación o por uso de subproductos para tráfico.

Considerando la magnitud de la problemática y el altísimo riesgo que significa para el mantenimiento de las poblaciones silvestres, invitamos a la comunidad científica a generar alianzas con las autoridades ambientales, y todos los actores relacionados con el tema, para participar en la generación de conocimiento en éste y demás temas que aporten a la conservación de grandes felinos, especies consideradas vulnerables en nuestro país.

\section{Referencias}

ACONCHA- ABRIL, I. En curso. Distribución del riesgo de depredación de animales domésticos por jaguar y puma en Colombia: herramientas para la planificación regional. Tesis para optar al Título de Magister en Manejo de Vida Silvestre. Universidad Nacional de Córdoba.

ARIAS-ALZATE, A., et al. 2011. Seguimiento del estado de las poblaciones de grandes felinos, así como la interacción de estos con los pobladores y sus actividades productivas en el Magdalena Medio Antioqueño. Informe Técnico. Universidad de Antioquia.

ARIAS-ALZATE, A., et al. 2012. Presencia de felinos y evidencias de conflicto con humanos en tres regiones de Antioquia. En Grandes Felinos de Colombia (Payan, E. \& C. Castaño-Uribe eds.). Bogotá, D. C.: Panthera Colombia, Conservación Internacional Colombia, Cat Specialist Group IUCN/SSC \& Fundación Herencia Ambiental Caribe.

ARIAS-ALZATE, A., et al. 2012a. Caracterización del estado de los felinos (Carnivora: Felidae) y su interacción con el hombre en el oriente de Antioquia. Documento técnico Corporación Autónoma Regional del Río Nare CORNARE.

AZEVEDO, F. C. \& D. L MURRAY. 2007. Evaluation of Potential Factors Predisposing Livestock to Predation by Jaguars. Journal of Wildlife Management 71: 2379-2386.

\footnotetext{
Mammalogy Notes | Notas Mastozoológicas

Sociedad Colombiana de Mastozoología

Vol. 3 Num. 1| 2016
} 
BOTERO-CRUZ, A. M. \& C. CASTAÑO-URIBE. 2007. Prospección y análisis del conflicto hombre - jaguar, en localidades de la zona amortiguadora del Parque Nacional Natural Paramillo - Córdoba. Informe Técnico. Plan de Conservación de félidos para el Caribe Colombiano \& Conservación Internacional Colombia.

CABRERA, J. A. 2013. Convenio de cooperación especial no. 200-12-17-461 entre la Corporación Autónoma Regional del Guavio y Fundación Panthera. Informe técnico. Fundación Panthera \& Corporación Autónoma Regional del Guavio CORPOGUAVIO.

CASTAÑO-URIBE C, et al. 2010. Guía de campo para la conservación de los felinos del Caribe colombiano. Felinos del Caribe: identidad biológica y cultural en una ecoregión felina. Ministerio de Ambiente, Vivienda y Desarrollo Territorial de Colombia, Conservación Internacional. Bogotá, Colombia.

CASTAÑN-URIBE, C., et al. 2013. Plan de Conservación de Felinos del Caribe colombiano: Los felinos y su papel en la planificación regional integral basada en especies clave. Fundación Herencia Ambiental Caribe, ProCAT Colombia, The Sierra to Sea Institute, Santa Marta, Colombia 232.

FERNÁNDEZ SALAZAR, A. C. 2012. Diagnóstico de la problemática que generan las especies de felinos con las comunidades humanas, con el fin de proponer estrategias de manejo en la jurisdicción de Corantioquia. Informe Final Corporación Autónoma Regional del Centro de Antioquia, CORANTIOQUIA.

GARCÍA-VILLAREAL, S. 2014. Revisión Sobre Estrategias de Manejo en Áreas Protegidas e Islas Frente a la Problemática de Perros Ferales (Canis lupus familiaris). Universidad Javeriana. Tesis de maestría. 45p.

GARROTE, G. 2012. Depredación del jaguar (Panthera onca) sobre el ganado en los llanos orientales. Mastozoología Neotropical, 19(1):139-145.

GONZÁLEZ-MAYA, J.F., 2010. Diagnóstico, evaluación y propuestas de solución a la problemática de conflictos ocasionados por Jaguar (Panthera onca) y Puma (Puma concolor) a actividades pecuarias en jurisdicción de la Corporación Autónoma Regional del Cesar-CORPOCESAR, departamento del Cesar, Colombia. 100 pp.

GONZÁLEZ-MAYA, J. F., et al. 2013. Conflictos felinos-vida silvestre en el Caribe colombiano: un estudio de caso en los departamentos del Cesar y la Guajira. En: Castaño-Uribe C, González-Maya J.F, Zárrate-Charry D., Ange-Jaramillo C. \& Vela-Vargas I.M. (eds). Plan de Conservación de Felinos del Caribe colombiano: Los felinos y su papel en la planificación regional integral basada en especies clave. Fundación herencia Ambiental Caribe, ProCAT Colombia, The Sierra to Sea Institute. Santa Marta, Colombia.

HIDALGO-JARAMILLO F. 2016. Exploring large felids-livestock-humans interactions and attitudes of local ranchers in the Caribbean region of Colombia. Tesis de maestría para optar el título de Manejo en Recursos sostenibles. Technische Universität München. 76 pp.

HOOGESTEIJN, R. \& A. HOOGESTEIJN. 2014. Anti-Predation Strategies for Cattle Ranches in Latin America: A Guide. Eckograf Solutions Publishers, Campo Grande, MS, Brazil.

INSKIP, C. \& A. ZIMMERMANN. 2009. Human-felid conflict: a review of patterns and priorities worldwide. Fauna \& Flora International, Oryx , 43(1): 18-34

MÁRQUEZ, R. \& I. GOLDSTEIN. 2014. Manual para el reconocimiento y evaluación de eventos de depredación de ganado por carnívoros silvestres. Versión 1.0.Wildlife Conservation Society Colombia. Santiago de Cali. pp. 35.MAVDT y FVSN. 2007. Programa Nacional de Conservación de Felinos de Colombia. Ministerio de Ambiente Vivienda y Desarrollo territorial; Fundación Vida Silvestre Neotropical. Bogotá, Colombia 81p

MAVDT \& FVSN. 2007. Programa Nacional de Conservación de Felinos de Colombia. Ministerio de Ambiente Vivienda y Desarrollo territorial; Fundación Vida Silvestre Neotropical. Bogotá, Colombia 81p.

MICHALSKI, F., et al. 2006. Human-wildlife conflicts in a fragmented Amazonian forest landscape: determinants of large felid depredation on livestock. Animal Conservation 9:179-188.

PANTHERA COLOMBIA \& CORMACARENA. 2011. Proyecto: estrategia de conservación de grandes felinos en el departamento del meta fase I. Informe técnico. Panthera Colombia \& CORMACARENA.

PAYÁN, E. 2004. Diagnóstico, análisis y propuestas de manejo para el conflicto de predación entre carnívoros y los sistemas productivos de la región Andina con énfasis en el eje cafetero de Colombia. Instituto Alexander von Humboldt, Bogotá, Colombia.

Mammalogy Notes | Notas Mastozoológicas

Sociedad Colombiana de Mastozoología

Vol. 3 Num. 1| 2016 


\section{Correspondencia | Correspondence}

PAYAN-GARRIDO, C. E. \& S. BORREGO 2005. Selección, tipificación y diseño de estrategias de manejo con fines antipredatorios y su sistema de seguimiento en predios pilotos de la región diagnosticada con ataques de felinos a sistemas ganaderos en el Eje Cafetero, Colombia. Instituto Alexander von Humboldt, Bogotá D. C., Colombia.

PAYÁN, E. 2005. Jaguar conservation in the Colombian Llanos: presence, local perceptions and the livestock conflict. Annual report, Jaguar Conservation Program, Wildlife Conservation Society, New York.

PINEDA-GUERRERO, A. \& D. A. ZÁRRATE- CHARRY 2014. Situaciones de conflicto por depredación y soluciones entre felinos y población local: una mirada a diferentes escalas. Boletín Aluna 5(1): 58-65.

PITMAN, M. R. P. L., et al. 2002. Manual de identificação, prevenção e controle de predação por carnívoros. Ediciones IBAMA. 83p.

QUIROZ, V. H. 2006. Diagnóstico de la situación actual de la depredación de ganado por felinos en jurisdicción de Corantioquia. Informe Final Corporación Autónoma Regional del Centro de Antioquia, CORANTIOQUIA.

RACERO-CASARRUBIA, J.A. 2007. Plan de monitoreo y manejo de jaguares (Panthera onca) que están generando conflictos con comunidades en la zona amortiguadora del PNN Paramillo, municipio de Tierralta - Córdoba. Informe Técnico. Unidad Administrativa Especial del Sistema de Parques Nacionales Naturales.

SALAZAR, E. et al. 2014. Factores de riesgo asociados al conflicto entre grandes felinos (Carnivora: Felidae) y humanos en Antioquia. Boletín Aluna 5(1): 53-57.

SARMIENTO, M. V. 2010. Patrón espacial de eventos de depredación de animales domésticos por jaguar (Panthera onca) y puma (Puma concolor) en los municipios de Hato Corozal y Tame, Orinoquia Colombiana. Tesis para optar el título de Master en Ciencias Biológicas. Universidad Nacional de Colombia sede Bogotá.

VELA-VARGAS, I. M et al. 2014. Diagnóstico y propuesta de mitigación a la problemática de conflictos ocasionados por Jaguar (Panthera onca) y Puma (Puma concolor) en la jurisdicción de la Corporación Autónoma Regional del GuavioCORPOGUAVIO, departamento de Cundinamarca. Proyecto de Conservación de Aguas y Tierras - ProCAT Colombia, The Sierra To Sea Institute, Corporación Autónoma Regional del Guavio - CORPOGUAVIO. Bogotá, Colombia. 98 pp.

ZÁRRATE CHARRY, D. A., et al. 2015. Caracterización y diagnóstico del conflicto en zonas vulnerables a la depredación a animales domésticos por parte de jaguares y pumas en el corregimiento de Siberia, Ciénaga-Colombia: Construcción de propuestas de mitigación. ProCAT Colombia, FUNDAHERENCIA, CORPAMAG. Santa Marta, Colombia. 43 pp.

ZÁRRATE-CHARRY, D., et al. 2010. Caracterización y diagnóstico de las poblaciones de félidos y otros mamíferos medianos y grandes en el departamento de la Guajira: estrategias de conservación a escala regional. Informe Técnico. Fundación Herencia Ambiental Caribe, Corporación Autónoma Regional de la Guajira-CORPOGUAJIRA, ProCAT Colombia. Santa Marta, Magdalena, Colombia. 51 pp.

ZIMMERMANN, A. et al. 2010. Contemporary views of human-wildlife conflicts on wild rangelands. Pp 129-151 In Wildrangelands: conserving wildlife while maintaining livestock in semi-arid ecosystems (J.T.d. Toit, R. Kock \& J.C. Deutsch eds.). Wiley-Blackwell, Chichester.

Mammalogy Notes | Notas Mastozoológicas

Sociedad Colombiana de Mastozoología

Vol. 3 Num. 1| 2016 


\title{
INFORMACIÓN SUPLEMENTARIA
}

\author{
Aconcha-Abril et al.
}

Bibliografía S1. Lista de los documentos relacionados con conflicto en Colombia.

1. ACONCHA- ABRIL, I. En curso. Distribución del riesgo de depredación de animales domésticos por jaguar y puma en Colombia: herramientas para la planificación regional. Tesis para optar al Título de Magister en Manejo de Vida Silvestre. Universidad Nacional de Córdoba.

2. ARIAS-ALZATE, et al. 2011. Seguimiento del estado de las poblaciones de grandes felinos, así como la interacción de estos con los pobladores y sus actividades productivas en el Magdalena Medio Antioqueño. Informe Técnico. Universidad de Antioquia.

3. ARIAS-ALZATE, A., et al. 2012. Presencia de felinos y evidencias de conflicto con humanos en tres regiones de Antioquia. En: Payan, E. \& C. Castaño-Uribe (eds.). Grandes Felinos de Colombia. Bogotá, D. C.: Panthera Colombia, Conservación Internacional Colombia, Cat Specialist Group IUCN/SSC \& Fundación Herencia Ambiental Caribe.

4. ARIAS-ALZATE, A., et al. 2012a. Caracterización del estado de los felinos (Carnivora: Felidae) y su interacción con el hombre en el oriente de Antioquia. Documento técnico Corporación Autónoma Regional del Río Nare CORNARE.

5. BOTERO-CRUZ, A. M. \& C. CASTAÑO-URIBE. 2007. Prospección y análisis del conflicto hombre - jaguar, en localidades de la zona amortiguadora del Parque Nacional Natural Paramillo - Córdoba. Informe Técnico. Plan de Conservación de félidos para el Caribe Colombiano \& Conservación Internacional Colombia.

6. CABRERA, J. A. 2013. Convenio de cooperación especial no. 200-12-17-461 entre la Corporación Autónoma Regional del Guavio y Fundación Panthera. Informe técnico. Fundación Panthera \& Corporación Autónoma Regional del Guavio CORPOGUAVIO.

7. CASTAÑO-URIBE, C., et al. 2013. Plan de Conservación de Felinos del Caribe colombiano: Los felinos y su papel en la planificación regional integral basada en especies clave. Fundación Herencia Ambiental Caribe, ProCAT Colombia, The Sierra to Sea Institute, Santa Marta, Colombia 232.

8. FERNÁNDEZ SALAZAR, A. C. 2012. Diagnóstico de la problemática que generan las especies de felinos con las comunidades humanas, con el fin de proponer estrategias de manejo en la jurisdicción de Corantioquia. Informe Final Corporación Autónoma Regional del Centro de Antioquia, CORANTIOQUIA.

9. GARCÍA-VILLAREAL, S. 2014. Revisión Sobre Estrategias de Manejo en Áreas Protegidas e Islas Frente a la Problemática de Perros Ferales (Canis lupus familiaris). Universidad Javeriana. Tesis de maestría. 45p.

10. GARROTE, G. 2012. Depredación del jaguar (Panthera onca) sobre el ganado en los llanos orientales. Mastozoología Neotropical, 19(1):139-145.

11. GONZÁLEZ-MAYA, J.F., 2010. Diagnóstico, evaluación y propuestas de solución a la problemática de conflictos ocasionados por Jaguar (Panthera onca) y Puma (Puma concolor) a actividades pecuarias en jurisdicción de la Corporación Autónoma

12. GONZÁLEZ-MAYA, J. F., et al. 2013. Conflictos felinos-vida silvestre en el Caribe colombiano: un estudio de caso en los departamentos del Cesar y la Guajira. En: Castaño-Uribe C, González-Maya J.F, Zárrate-Charry D., AngeJaramillo C. \& Vela-Vargas I.M. (eds). Plan de Conservación de Felinos del Caribe colombiano: Los felinos y su papel en la planificación regional integral basada en especies clave. Fundación herencia Ambiental Caribe, ProCAT Colombia, The Sierra to Sea Institute. Santa Marta, Colombia.

13. HIDALGO-JARAMILLO F. 2016. Exploring large felids-livestock-humans interactions and attitudes of local ranchers in the Caribbean region of Colombia. Tesis de maestría para optar el título de Manejo en Recursos sostenibles. Technische Universität München. 76 pp.

14. MÁRQUEZ, R. \& I. GOLDSTEIN. 2014. Manual para el reconocimiento y evaluación de eventos de depredación de ganado por carnívoros silvestres. Versión 1.0.Wildlife Conservation Society Colombia. Santiago de Cali. pp.

\footnotetext{
Mammalogy Notes | Notas Mastozoológicas

Sociedad Colombiana de Mastozoología

Vol. 3 Num. 1| 2016
} 
35.MAVDT y FVSN. 2007. Programa Nacional de Conservación de Felinos de Colombia. Ministerio de Ambiente Vivienda y Desarrollo territorial; Fundación Vida Silvestre Neotropical. Bogotá, Colombia 81p

15. MAVDT \& FVSN. 2007. Programa Nacional de Conservación de Felinos de Colombia. Ministerio de Ambiente Vivienda y Desarrollo territorial; Fundación Vida Silvestre Neotropical. Bogotá, Colombia 81p.

16. PANTHERA COLOMBIA \& CORMACARENA. 2011. Proyecto: estrategia de conservación de grandes felinos en el departamento del meta fase I. Informe técnico. Panthera Colombia \& CORMACARENA.

17. PAYÁN, E. 2004. Diagnóstico, análisis y propuestas de manejo para el conflicto de predación entre carnívoros y los sistemas productivos de la región Andina con énfasis en el eje cafetero de Colombia. Instituto Alexander von Humboldt, Bogotá, Colombia.

18. PAYAN-GARRIDO, C. E. \& S. BORREGO 2005. Selección, tipificación y diseño de estrategias de manejo con fines antipredatorios y su sistema de seguimiento en predios pilotos de la región diagnosticada con ataques de felinos a sistemas ganaderos en el Eje Cafetero, Colombia. Instituto Alexander von Humboldt, Bogotá D. C., Colombia.

19. PAYÁN, E. 2005. Jaguar conservation in the Colombian Llanos: presence, local perceptions and the livestock conflict. Annual report, Jaguar Conservation Program, Wildlife Conservation Society, New York.

20. PINEDA-GUERRERO, A. \& D.A. ZÁRRATE- CHARRY 2014. Situaciones de conflicto por depredación y soluciones entre felinos y población local: una mirada a diferentes escalas. Boletín Aluna 5(1): 58-65.

21. QUIROZ, V.H. 2006. Diagnóstico de la situación actual de la depredación de ganado por felinos en jurisdicción de Corantioquia. Informe Final Corporación Autónoma Regional del Centro de Antioquia, CORANTIOQUIA.

22. RACERO-CASARRUBIA, J.A. 2007. Plan de monitoreo y manejo de jaguares (Panthera onca) que están generando conflictos con comunidades en la zona amortiguadora del PNN Paramillo, municipio de Tierralta - Córdoba. Informe Técnico. Unidad Administrativa Especial del Sistema de Parques Nacionales Naturales.

23. SALAZAR, E. et al. 2014. Factores de riesgo asociados al conflicto entre grandes felinos (Carnivora: Felidae) y humanos en Antioquia. Boletín Aluna 5(1): 53-57.

24. SARMIENTO, M. V. 2010. Patrón espacial de eventos de depredación de animales domésticos por jaguar (Panthera onca) y puma (Puma concolor) en los municipios de Hato Corozal y Tame, Orinoquia Colombiana. Tesis para optar el título de Master en Ciencias Biológicas. Universidad Nacional de Colombia sede Bogotá.

25. VELA-VARGAS, I. M et al. 2014. Diagnóstico y propuesta de mitigación a la problemática de conflictos ocasionados por Jaguar (Panthera onca) y Puma (Puma concolor) en la jurisdicción de la Corporación Autónoma Regional del Guavio-CORPOGUAVIO, departamento de Cundinamarca. Proyecto de Conservación de Aguas y Tierras - ProCAT Colombia, The Sierra To Sea Institute, Corporación Autónoma Regional del Guavio CORPOGUAVIO. Bogotá, Colombia. 98 pp.

26. ZÁRRATE CHARRY, D.A. et al. 2015. Caracterización y diagnóstico del conflicto en zonas vulnerables a la depredación a animales domésticos por parte de jaguares y pumas en el corregimiento de Siberia, Ciénaga-Colombia: Construcción de propuestas de mitigación. ProCAT Colombia, FUNDAHERENCIA, CORPAMAG. Santa Marta, Colombia. 43 pp.

27. ZÁRRATE-CHARRY D. et al. 2010. Caracterización y diagnóstico de las poblaciones de félidos y otros mamíferos medianos y grandes en el departamento de la Guajira: estrategias de conservación a escala regional. Informe Técnico. Fundación Herencia Ambiental Caribe, Corporación Autónoma Regional de la Guajira-CORPOGUAJIRA, ProCAT Colombia. Santa Marta, Magdalena, Colombia. 51 pp. 